\title{
Closing the gap: astrocytes and brain metastasis
}

\author{
Cell Research (2016) 26:973-974. doi:10.1038/cr.2016.96; published online 12 August 2016
}

Astrocytes are emerging as essential regulators of brain metastasis progression. In a current issue of Nature, Chen et al. identify a novel mechanism of astrocyte-carcinoma interaction and exploit vulnerabilities therein to slow brain metastatic growth in pre-clinical models.

Brain metastasis is a serious obstacle in cancer patient care and a significant indicator of dismal prognosis. Their high incidence makes them the most prevalent intracranial malignancy. In the majority of cases, treatment is palliative and most patients succumb to disease progression on the order of months after diagnosis. The knowledge gained from clinical assessment is hampered by the exclusion of patients with symptomatic brain metastasis (BM) from clinical trials, and preclinical research has been hindered by the lack of translational efficacy.

Despite these challenges, a number of preclinical studies over the last several years have provided important insights into the biology of BM [2]. In one example, Dr Massagué and colleagues identified the sialyltransferase ST6GALNAC5 as a critical regulator of cancer cell extravasation into the brain parenchyma [3]. Once extravasated, successful colonization of brain metastases depends on persistent contact with microvessels [4]. Consistent with these findings, a key mediator of vessel co-option, the cell adhesion molecule L1CAM, was identified as a requirement for cancer cell survival and brain metastasis initiation [5]. Once infiltrated into the brain tissue, cancer cells encounter a number of host cell types, including pericytes, reactive glia, neural progenitor cells, neurons and oligodendrocytes. Analysis of breast cancer BM shows an abundance of activated astrocytes and microglia around and within the lesion [6]. The role of these cells within BM is subject of intensive investigation. Recently, Massagué and colleagues discovered that cancer cells must express serpin protein family members to survive the initial FasL-mediated apoptosis regulated by astrocytes [5].

In a recent issue of Nature, Chen et al. [1] build upon this work to describe novel mechanisms set in motion when tumors expand in the brain parenchyma. Astrocytes play a key role in this process (Figure 1). The authors tease out the pro-survival communication between astrocytes and cancer cells. Breast and lung cancer cells that express protocadherin 7 (PCDH7) promote tumorastrocyte gap junction formation by recruiting connexin $43(\mathrm{Cx} 43)$. These channels allow passage of cGAMP from cancer cells to astrocytes triggering the secretion of inflammatory cytokines via STING pathway activation. This cascade activates STAT1 and NF- $\kappa$ B pathways in cancer cells promoting tumor growth and resistance to chemotherapy.

Recapitulation of the BM cascade in animal models is challenging. Chen et al. utilize a validated model of intracardiac injection of breast or lung cancer cells selected for their brain tropism. This model allows the study of extravasation and progression into the brain microenvironment. Extravasation of circulating tumor cells into the brain parenchyma triggers a rapid response from the stroma. Glial cells, including astrocytes and microglia, display a dynamic interaction with tumor cells reminiscent of brain injury response. Astrocytes are key regulators of the blood-brain barrier (BBB) and form a glial scar following a breach and lesion of the brain parenchyma. In separate publications, Massagué and colleagues demonstrate two seemingly opposing roles for astrocytes in brain metastasis: eliminating cancer cells entering the brain parenchyma versus promoting metastatic progression in the brain. It seems that the cancer cells surviving the initial attack from astrocytes can benefit from the pro-survival signals emanating from the attackers. Chen at al. show that this occurs via the formation of gap junctions between activated astrocytes and cancer cells. The observation that cancer cells and astrocytes communicate through gap junctions is consistent with other findings on the exchange of pro-survival signals and ions through gap junctions as a potential mechanism of resistance to chemotherapy in breast, melanoma, and lung cancer cells [7, 8]. BM progression is also facilitated via downregulation of tumor PTEN by miRNA-containing exosomes secreted by astrocytes [9]. This complexity of the astrocyte-cancer cell interaction will likely require attacking multiple nodes to improve therapeutic outcome.

The resistance of brain lesions to therapies, which are effective in extracranial sites, represents a formidable barrier in oncology. Progression of $\mathrm{BM}$ in the face of various treatments has been attributed to poor delivery due to the BBB and/or cell-intrinsic resistance mechanisms. Chen et al. demonstrate that $\mathrm{Cx} 43$ and PCDH7 depletion (via shRNA or pharmacologi- 


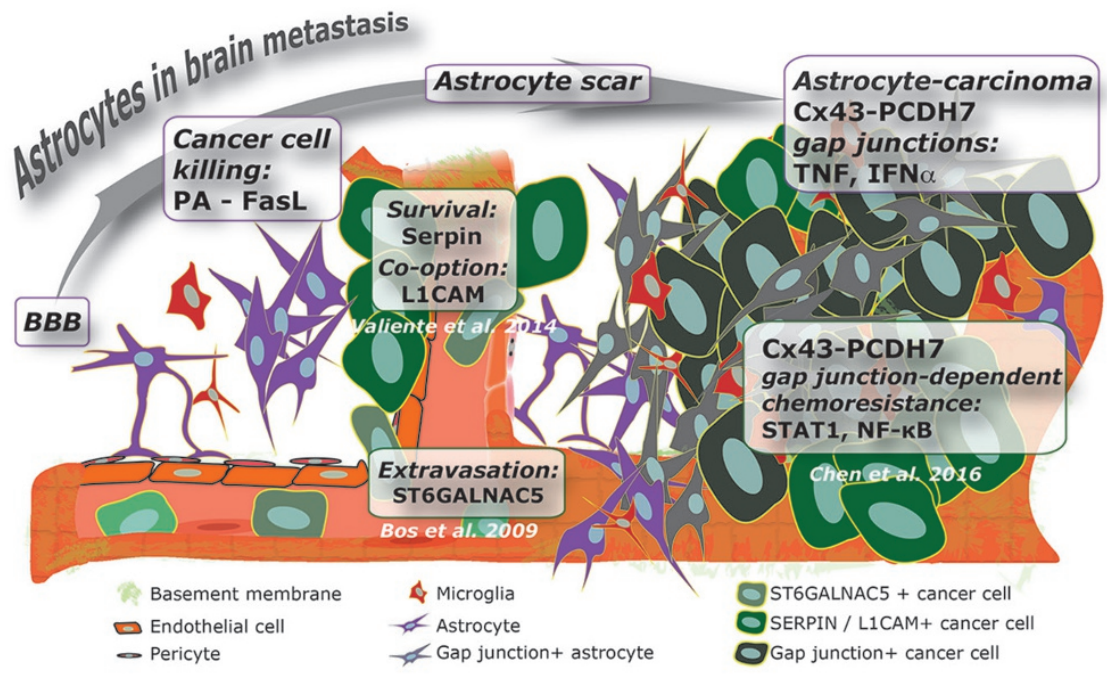

Figure 1 Astrocytes impact both early and late stage brain metastasis progression. Circulating tumor cells producing ST6GALNAC5 bypass the BBB and seed the brain parenchyma. Tumor L1CAM and SERPIN expression is required for colonization and expansion in the brain. This sequence of events suggests that the astrocyte response selects for cancer cells with the best survival potential in the brain microenvironment. Chen et al.'s findings reveal that enduring cancer cells in turn instruct astrocytes to promote survival signaling.

cal inhibition) overcomes resistance to the BBB-penetrant carboplatin and reduces tumor burden compared to monotherapy. Importantly, shRNAmediated knockdown of $\mathrm{CX} 43, \mathrm{PCDH7}$ or STAT1, and knockdown of Sting is sufficient to significantly reduce tumor burden compared to controls. Furthermore, Chen et al. confirm that $\mathrm{Cx} 43$ gap junction-dependent mechanisms are shared between breast and lung cancer brain metastases. In some ways, this comes as no surprise because the brain microenvironment is known to reprogram breast, lung and melanoma cells [10]. Focusing on the tumor-brain microenvironment and cancer cellextrinsic mechanisms of resistance may lead to novel strategies to treat brain metastasis as a single disease entity regardless of the type and subtype of disease. Moreover, it is likely that targeted therapies could also benefit from these findings. The antibody-chemotherapy conjugate ado-trastuzumab emtansine (T-DM1) reduces BM progression in pre-clinical models of HER2-positive breast cancer when compared to conventional therapies such as trastuzumab [11]. It would be reasonable to expect a synergistic effect with the combination of astrocyte-carcinoma gap junction inhibitors and T-DM1 when treating HER $2^{+}$breast cancer BM. These therapies could also be potentially combined with checkpoint blockers, as recently demonstrated for T-DM1 and CTLA4/ PD-1 blockade [12].

Finally, Chen et al. provide expression analyses in matched clinical specimens that support the translational value of their pre-clinical findings. Analysis of clinical cohorts demonstrated that the expression of PCDH7 and CX43 in primary tumors correlates with increased brain metastasis in TNBC and decreased metastasis-free survival in NSCLC. These molecules could serve as potential biomarkers to assess the risk of brain metastasis in patients, and to inform their treatment. The findings offer new hope in the treatment of brain metastasis by highlighting key components of a "pro-survival" metastatic cascade. Chen et al. show that $\mathrm{PCDH} 7$ and $\mathrm{Cx} 43$ depletion reduces late stage progression and not early extravasation and vascular co-option processes. Together, strategies disrupting astrocyte-cancer cell interactions has the potential to improve both prevention and treatment of brain metastasis.

Gino B Ferraro ${ }^{1}$, David P Kodack ${ }^{1}$, Vasileios Askoxylakis ${ }^{1}$, Rakesh K Jain ${ }^{1}$

${ }^{1}$ Edwin L. Steele Laboratory, Department of Radiation Oncology, Massachusetts General Hospital and Harvard Medical School, Boston, MA 02114, USA

Correspondence: Rakesh K Jain

E-mail: jain@steele.mgh.harvard.edu

\section{References}

1 Chen Q, Boire A, Jin X, et al. Nature 2016; 533:493-498.

2 Kodack DP, Askoxylakis V, Ferraro GB, et al. Cancer Cell 2015; 27:163-175.

3 Bos PD, Zhang XH, Nadal C, et al. Nature 2009; 459:1005-1009.

4 Kienast Y, von Baumgarten L, Fuhrmann M, et al. Nat Med 2010; 16:116-122.

5 Valiente M, Obenauf AC, Jin X, et al. Cell 2014; 156:1002-1016.

6 Zhang M, Olsson Y. J Neurol Sci 1995; 134:26-32.

7 Kim SJ, Kim JS, Park ES, et al. Neoplasia 2011; 13:286-298.

8 Lee HJ, Hanibuchi M, Kim SJ, et al. Neuro Oncol 2016; 18:486-496.

9 Zhang L, Zhang S, Yao J, et al. Nature 2015; 527:100-104.

10 Park ES, Kim SJ, Kim SW, et al. Proc Natl Acad Sci USA 2011; 108:17456-17461.

11 Askoxylakis V, Ferraro GB, Kodack DP, et al. J Natl Cancer Inst 2015; 108:djv313.

12 Müller P, Kreuzaler M, Khan T, et al. Sci Transl Med 2015; 7:315ra188. 